\title{
Automatic discrimination of Costa Rican stingless bees based on modified SIFT of its wings
}

\author{
Juan Pablo Prendas Rojas \\ Mathematics Department \\ Costa Rica Institute of Technology (TEC) \\ Cartago, Costa Rica \\ jpprendas@itcr.ac.cr \\ Melvin Ramirez Bogantes \\ Mathematics Department \\ Costa Rica Institute of Technology (TEC) \\ Cartago, Costa Rica \\ meramirez@itcr.ac.cr \\ Ingrid Aguilar Monge \\ Centre for Tropical Bee Research (CINAT) \\ National University of Costa Rica \\ Heredia, Costa Rica \\ ingrid.aguilar.monge@una.cr
}

\author{
Geovannie Figueroa Mata \\ Mathematics Department \\ Costa Rica Institute of Technology (TEC) \\ Cartago, Costa Rica \\ gfigueroa@itcr.ac.cr
}

\author{
Carlos Travieso Gonzalez \\ Institute for the Technological Development and \\ Communications Innovation (IDeTic) \\ Las Palmas de Gran Canaria University \\ carlos.travieso@ulpgc.es \\ Eduardo Herrera Gonzalez \\ Centre for Tropical Bee Research (CINAT) \\ National University of Costa Rica \\ Heredia, Costa Rica \\ apieduardo@gmail.com
}

\begin{abstract}
The creation of an automated system based on digital image processing of photographs of their wings to identify the genus and species of a bee (Apidae: Meliponini) is proposed. A fast and efficient system of taxonomic classification of stingless bees would be a very valuable contribution, as well as a reference for the study of stingless bees. The fore and hind right wings of workers were placed in an inert medium. The bee samples were collected directly from their nests and from the entomological collection of the Tropical Bee Research Center (CINAT).

Three collections of bees were created at CINAT; the first one is about individuals bees, the second is actual bee wings preserved into a specialized balsam and the last one is a collection of photos from the bee wings. In total material was processed for $\mathbf{2 0}$ genera (90\% of genera of native stingless bees present in Costa Rica) and 59 species $(73 \%$ of the species reported).

Automatic discrimination system used a dataset by 459 images, grouped into 18 species of 10 genera. After to applying the matching approach, the specie accuracy reached an $86.49 \%$, and the genera accuracy was $\mathbf{9 7 . 5 2 \%}$.
\end{abstract}

Keywords - stingless bees; venation classification; SIFT; image processing

\section{INTRODUCTION}

Bees constitute a diverse and important group of insects for the dynamics of forest and agro ecosystems [1]. About 20,000 species are known, of which most of them have solitary habits [2] so it complicated to study them and propose conservation strategies. One of the main problems for native bee conservation in Latin America is the absence of important information about the richness, diversity, taxonomy, distribution, population dynamics and the impact of human activities on all species of bees [3]. These topics have not been studied extensively in the American tropics. Therefore, it is proposed the creation of collections (physical and digital) and an automatic system for the stingless bees identification.

More specifically, the native stingless bees are a very important component in the natural tropics and subtropics ecosystems of the world because they facilitate pollination of most crop plants [4]. Over 400 species are known, of which 300 are in the tropics and subtropics of America. In Costa Rica there are almost 20 genera and 59 species of stingless bees [5], among which the most important is the new specie described recently (Nogueirapis costaricana) [6]. The stingless bees have been an essential part of Hispanic culture and nowadays are being used in a wide variety of projects focused on the family economy, forest conservation and community tourism. Its principal products are honey, propolis and pollen. These products are highly prized among the local population for their own antimicrobial properties [7].

Some developmental issues regarding the taxonomy of bees in Costa Rica, especially in Meliponini specie, are the lack of appropriate entomological collections covering the whole country stocks as well as guides for their classification available to all public. There is a need to search for quick and easy systems to address doubts about species identification. This is increasingly evident in remote areas where experts cannot always do their jobs [8]. An alternative solution to this 
problem would be a digital system for bee identification, which can be easy to use and has reliable results.

\section{RELATED WORKS}

With the development of technology and the growth in processing capability of computers, very efficient systems have been proposed for automatic classification of bees from the wings images. Studies show that the wings contain important information for insect identification; since they vary more between different sub-species within the same species and yet differences allow discrimination between different species [9] [10]. The insect wings are membranous with visible veins, which allow the creation of a clear diagram. In [11-13] it is mentioned that the classification of insects based on the characteristics of the venation of the wings has been very successful in recent years, as the use of morphometric indicators have gained importance in biological areas.

The first proposal for an automatic system for bee classification was in the early 80's with the creation of Fast Africanized Bee Identification System (FABIS). There is not much information available about this system, however in [14] it is indicated that the FABIS could accurately classify Africanized bees. For discrimination of bees it needs to take 25 morphometric measurements, requiring a training process and availability of several hours for the calculations. For that reason, the system has been discontinued, but the automatic bee identification initiative has continued.

Without a doubt, the most recognized contribution to the problem of automating bee discrimination has been the Automatic Bee Identification System (ABIS). ABIS was proposed in 1991 and was used until 2008, unfortunately after this year it has been discontinued. ABIS's main strategy was to use wing information similar to the use of the fingerprint in humans. This used a large database (300 characteristics) for further statistical recognition.

The cells formed inside the wings receive particular attention. With the information in venation lines and their intersections, the system generates hypotheses about the location of the cells. The available information about the results obtained with ABIS indicates a 99.8\% recognition success at genera level. However, it is important to note that only the following genera were resolved: Colletes, Andrea y Bombus. At species level, in those genera, success decreased to $95 \%$.

Although the strategy of ABIS system achieved a high success rate, it has some limitations among which are the use of very few species and genera, it demands a training system with at least 20 specimens per class (either species or genera) and requires that the user manually identify some characteristics.

Due to the success of this system recently some proposals have emerged which aim to elucidate the recognized limitations in ABIS. In 2001, there was a publication that highlights the accuracy, portability and tools used by ABIS, but identified as the creation of public access, monitoring the system and the incorporation of geographic information [8].
More recently, in 2008, the possibility of combining the results generated by the ABIS system with ties to morphometric descriptors present in the wings was proposed [17]. This proposal prompted the use of complementary software in processes discrimination. In photographs, after being processed in ABIS, 19 homologous intersections of venations are marked using the software tpsDig and these points were aligned and compared using analysis of Procrustes. ABIS combined with morphometric analysis gave accurate results of $98.05 \%$ in Africanized and $80 \%$ with the inclusion of the other four species.

Finally, in 2011 the MelittO Biotaxis System (MOBS) was proposed in order to identify live bees by anatomical characteristics of their wings [11]. MOBS had an efficiency of $90 \%$ in the identification of 16 species grouped into four genera. What is really innovative in MOBS is the automatic reduction of the characteristics used in the discrimination (under 20) compared to ABIS. In a first step, the wing venation is extracted (using mathematical morphology and thresholds), the cells are extracted and tagged (as in ABIS), extraction of vein crossings and vein tagging; in a later phase, techniques (including PCA, SIFT and SURF) are combined to select the most important characteristics. In [11], we conclude that veins crosses crossings and centres of mass of the wings are excellent key points and also indicate that a poor training would imply classification problems.

Up to this point it has been shown that there are several systems capable of classifying species of bees efficiently; however we can also list some important limitations. First, none has been used for discrimination of stingless bees, few species and genera were used, most systems are not fully automatic (requiring active user intervention), some of them require many hours of training and perhaps the most relevant, they are designed to classify a group of images.

For these reasons, it was considered important to create a system of discrimination of stingless bees from a photograph of the wing. An easy to use system is proposed where only a photograph need be entered and the system determines to what species the wing belongs.

\section{DISCRIMATION SYSTEM BASED ON SIFT}

The process for creation of a automatic discrimination system of bee's species needed four steps:

\section{A. Creating Database}

Creating database includes preparation of the material for three collections; bee specimens, another front and rear right wing mounted in an inert preservation environment and a third collection of photographs of plates.

The bees were collected directly from nests in different geographical areas of Costa Rica, using entomological and sniffers network. A 'death camera' was used with ethyl acetate in order to kill quickly and avoid damage to the wings. The samples were transported to CINAT where the mounting on entomological pins and the respective identification were made. This collection has a total of 879 examples. 
Then, some bee wings were removed from their mesatorax whit a razor blade and dissecting scissors to facilitate removal and avoid damaging them. The collection of wings has a total of 879 plates. An Olympus model SZX16 stereoscope was used for taking images with an integrated camera. The resolution in the millimetre was $2 \mathrm{x}$ with a focal opening of $1 / 4$. The generated images were stored in a database, grouped by species. The collection of photographs has a total of 1758 pictures.

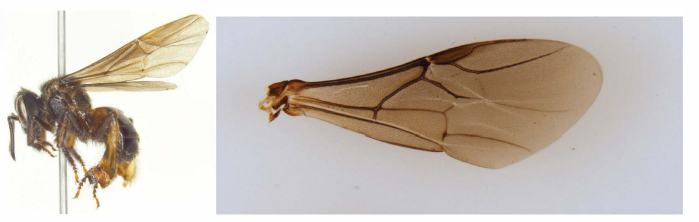

Fig. 1. (a) Specimen of Trigona nigerrima mounted on an entomological pin. (b) Fore wing image generated from the capture protocol of the wings

\section{B. Back ground Substraction}

There are many different techniques to distinguish whether a pixel belongs to the background of the image [15-17]; in this research it used mainly histograms, thresholds and morphological operators.

In the collection of photographs of bees discrimination between two backgrounds was achieved; one with grey and one with presence of blue. This required that the algorithm, initially determine which type of background had the picture, because this first decision would depend on the application of Otsu method and threshold settings. For this purpose, the space with colour HSV and one empirically calculated dimension was used to determine which of the backgrounds the photo had. Once the type of background was identified, a filter was implemented by experimentally adjusted threshold (Otsu) to determine which pixels will become white and which in blacks. In Figure 2, the blank region of the second picture corresponds to what was identified as wing and the last image is the recovery of the original colour in the points with allocation 'white'.
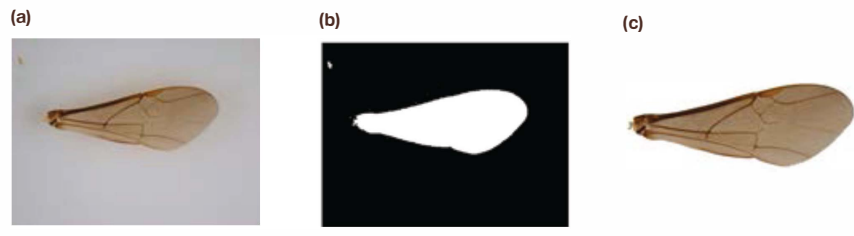

Fig. 2. (a) Sample of original photo of the collection, (b) photograph morphological operations, (c) result of the elimination of background

Pictures with the presence of two wings had an additional problem that occurred in the process of eliminating background. During the protocol of taking the photograph, it was decided to focus on the wing of interest to make this the discriminator element in which there were two or more wings.

To solve this problem, the calculation of the center of mass of all resulting white regions was included in the background removal algorithm (if there is more than one wing there should be more of a white region). Also included was measurement of the distances of these points to the center of the image, because the shortest distance will discriminate the white region that corresponds to the wing of interest. An illustration of this strategy is shown in Figure 3.

\section{Parameterization SIF}

The Scale Invariant Feature Transform (SIFT) is a method proposed by David Lowe in 1999 and explicitly exposed in 2004. The basic idea is the transformation of an image to a representation composed of "points of interest" [18-19]. This setting has been used in diverse areas such as security, face recognition, object recognition, among others. However, and this must be highlighted, its use has largely diffused recognition of same individual or identical object [20-21] so that implementation of the SIFT for discrimination of insect species is new and must be done with special care for natural variants of individuals even of the same species.

In that sense, the choice of the SIFT parameterization is based on the largest number of key points that generates and lower processing cost, in comparison with SURF and ASIFT e.g. [21-22]. Since the pictures do not correspond to the same individual it was considered important to generate a large number of key points, which describe each image and then be exhaustive in the matching of these points.
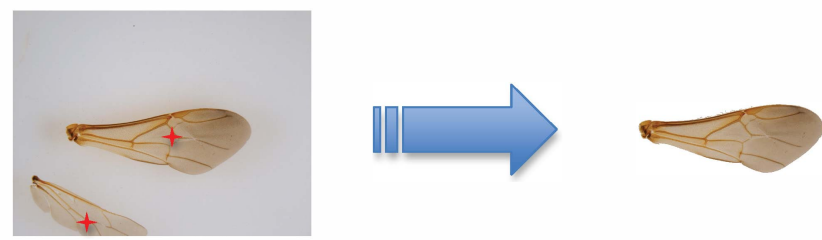

Fig. 3. Selection of wing of interest from the nearness of center of the picture with the center of mass of the wing

The SIFT method is summarized by the following phases:

Construction of pyramids of Scale-Space. The reduction image to a set of parameters invariant to scale and rotation is achieved by creating pyramids where the scale of the photograph is changing and there is convolution with a Gaussian function [23-25].

The image obtained by the difference of Gaussians highlights the edges and corners of the image, as shown in Figure 4. Since the available images are large (1920 x 2560), the illustration was calculated with a Gaussian with a high $\sigma$ value and a square convolution matrix of order 50 .

Location of key points. When the Gaussian differences are already calculated the most of points in the image are cero. Candidates to key points should be identified between the higher and lower values of points different to zero. It is important that the comparison of each point with its neighbors is also made in the next scale. Then these are discriminated by stability [23]. 

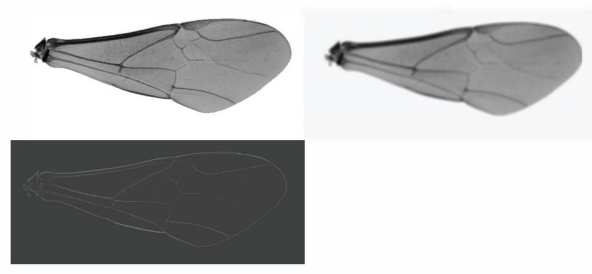

Fig. 4. Illustration of the effect of Gaussian filtering and Gaussian difference.

Orientation assignment. To assign orientation to the points ( $x$, $y$ ) referred keys in the previous phase; the gradient magnitude and orientation are used [23-24].

Descriptor of key points. There is assigned to each key point a set of characteristics among which stand out the position, orientation and rotation of the gradient (to achieve invariance about the rotation in $2 \mathrm{D}$ or $3 \mathrm{D}$ or changes in viewpoints in 3D). Each point has an associated matrix of $4 \times 4 \times 8$, i.e. 128 characteristics [20-21]. The Figure 5 shows the SIFT output for a wing image with a lot of key points.

Matching. Establish if there is correspondence between the characteristics of a key point to the characteristics of any of the other image, the Euclidean distance is used. This strategy does not rule out the possibility of multiple, incorrect and no correspondence matching, however it was considered better to have sufficient correlation (even if they are incorrect) with SIFT outputs and then refine assignments, so the threshold used in the matching was relaxed slightly.

\section{New Parameter Included on Matching SIFT}

Discriminator parameters usually introduced in the SIFT matching was insufficient because in many cases the matching assignments were made incorrectly. In this situation it would be not possible to determinate the magnitude of bad allocations. Figure 6 shows a case where the matching was incorrect for a large number of key points. Although it could be argued that the photographs are not adequate regarding the protocol capture parameters used in the preprocessing, the fact is that this case is chaotic and suggests the need to incorporate a more robust discriminator for the matching phase.

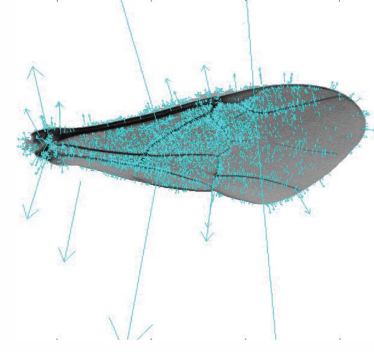

Fig. 5. Out of the SIFT parameterization for a photo with 4477 key points.

It is also necessary that the new discriminator can be invariant to image size and rotation conditions because these conditions are set by the SIFT method. It was decided to incorporate a correspondence discriminator, which is a little more demanding. A pair of key points identified as corresponding in the output SIFT must also measure smaller than a value $\lambda$ that is updated for each assignment.

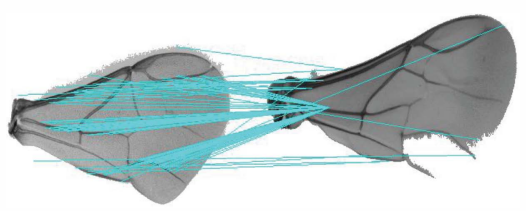

Fig. 6. An extreme case of incorrect correspondences between the species Cephalotrigona zexmeniae and Melipona beechei

For a key point with vector director $\left(x_{1}, y_{1}\right)$ and another key point $\left(x_{2}, y_{2}\right)$ in another image, consider

$$
\begin{gathered}
r_{1}^{2}=\left(x_{1}-x_{1 c}\right)^{2}+\left(y_{1}-y_{1 c}\right)^{2} \\
r_{2}^{2}=\left(x_{2}-x_{2 c}\right)^{2}+\left(y_{2}-y_{2 c}\right)^{2} \\
\rho_{1}=\frac{r_{1}}{A_{1}} \quad \rho_{2}=\frac{r_{2}}{A_{2}} \\
\left|\rho_{1}-\rho_{2}\right|<\lambda
\end{gathered}
$$

Where the ordered pair $\left(x_{1 \mathrm{c}}, y_{1 \mathrm{c}}\right)$ are the coordinates of the center of mass of the wing of the image where $\left(x_{1}, y_{1}\right)$ is the key point and in analogy for $\left(x_{2 \mathrm{c}}, y_{2 \mathrm{c}}\right)$. The constants $A_{1}$ and $A_{2}$ are the areas of the corresponding wings.

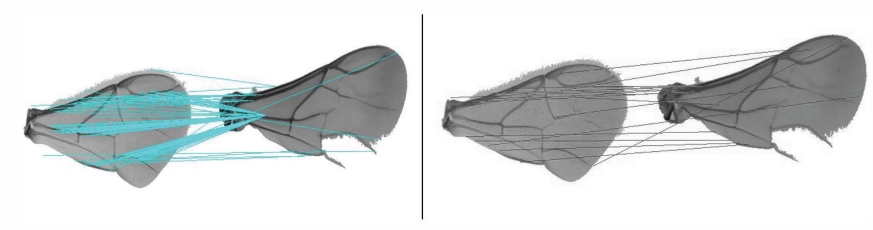

Fig. 7. Effect of the incorporation of the new descriptor in the correlation between the species Cephalotrigona zexmeniae y Melipona beecheii

The value is a measure of the difference between the parameters described in Equation (1). The parameters are normalized ratios measured from the vector director of the key point to the center of mass of the wing. In order to normalize the Euclidean distance a quotient for the wing area was used. With the incorporation of this discriminator the invariance regarding the scale and rotation is ensured and also the assignments where the points have very different positions with respect to the centers of mass of the wings are discarded. Figure 7 shows the improvement in a case where there had been many incorrect assignments.

\section{E. Discrimination of species}

Starting from a database with photographs properly processed in the background elimination phase and in collaboration with biologists specializing in bee studies, a photograph of each species was selected to create a reference base for the system. The photos were selected carefully assuring they were a good representation of the species, from 
the biological point of view, and that previous preprocessing phases had been perfectly successful [26]. In other words, besides to achieving the form, color and texture requirements, a photo should be such that the wing can be studied without noise and the information it contains is only about the wing.

The proposed system (illustrated in Figure 8) receives a $\mathrm{Im}$ image which contains a wing (or two) of a Costa Rican native bee. Then it is processed to create another Imp image in which the background has been removed. The next step is the application of the SIFT parameterization to the new Imp photo, and then a matching with each of the photos in the reference base. The system saves the number of correspondences obtained with each In image in the base, with this information the maximum of these correspondences is calculated.

If the maximum of correspondence occurred with the $I k$ image then the photo received by the system discriminates as the species tagged in the image at position $k$. Clearly some situation could occur where the system cannot decide, if the maximum correlation appears in several images or if it is only in one species but its value is too small to be considered a successful discrimination. These situations are accounted for in the system: this would report automatically that there are 'multiple matches' or that they are 'insufficient coincidences'. For the last situation, a minimum of experimental coincidences $N$ of 25 key points has been established.

The algorithm was programmed in MatLab and the vlfeat library (available in http://www.vlfeat.org/index.html) was used for the implementation of SIFT and assignments correlation.

\section{RESULTS}

The strategy for the collection and analysis of results was to work with a small sample of images in which the processing of background removal were calibrated, along with SIFT parameterization and determination of correspondences; then the algorithm is trained on a larger sample and finally the calculations were validated in another sample totally independent of those images used in the earlier phases.

Table 1 shows a portion of the cross-matrix discrimination. In the row labels the tags that identify the images present in the sample are placed and in the column labels are the reference images. The algorithm calculated for each image all matches with reference images.

In order to provide clarity to the process the places where the maximum correlation should be obtained were highlighted with colored cells and the maximums were highlighted in red. If the red number is in a position, which does not correspond with the highlighted color, the result is considered a wrong. For example, all images of Geotrigona lutzi (G. lu) species were discriminated perfectly while 3 images of Dolichotrigona schulthessi (D. sc) species were assigned to the wrong kind of specie.

The implementation of the algorithm in the training phase had a really good success rate but it was considerably far to $100 \%$. The parameters were adjusted and the validation phase began in an independent sample of photographs.

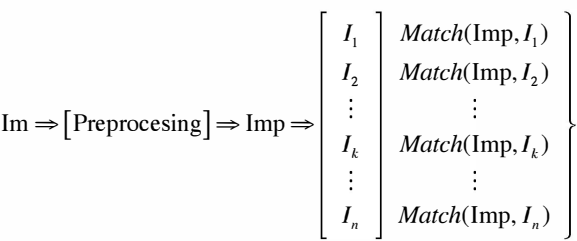

$$
\begin{aligned}
& M_{k}=\max (\text { Match }) \Rightarrow\left\{\begin{array}{l}
M_{k} \text { is unique }\left\{\begin{array}{l}
M_{k} \geq N \Rightarrow \text { The bee in the photo belong to specie k } \\
M_{k}<N \Rightarrow \text { Insufficient Matches }
\end{array}\right. \\
M_{k} \text { is not unique } \Rightarrow \text { Multiple Maches }
\end{array}\right.
\end{aligned}
$$

Fig. 8. Scheme processing photo of bee wing, matching with database photos and final decision about the species present in the photo

A sample of 459 photographs grouped into 18 species in 10 genera was used to validate the results. It is important to clarify that the time allocated for this project did not allow sufficient collection of specimens from the remaining 7 species to cover the stages of training and validation. Therefore, although the algorithm is trained with 25 species, the validation stage can only include 18 . This is not alarming because of the references consulted none have reached that number of species discriminated.

The Table 2 shows information about the 18 species (name, label, sample size for each specie, number of wrong matches) and the use of weighted average for calculating the success percent about species (86.49) and genera (97.52).

\section{CONCLUSIONS AND FUTURE TASK}

In the discrimination system it is expected that the success rate is close to 100 . With genus discrimination of native stingless bees this expectation has been fulfilled, however the percentage of identification of each species needs to improve. This task has already begun [27], as of this writing quantifiable data is not yet available but it is possible to offer some ideas.

Based on the information provided by expert biologists in apiculture studies, it was decided to incorporate some species discriminators that are not in the wing, such as colors from other parts of the body, downiness, size of their legs, antennae or others. The proposal is to ensure that the system when has any 'doubts' about the species, either by the existence of very close to the maximum of matches or multiple matches values, it becomes a semi-aided system.

For example, it has been determined that the following species Trigona silvestriana and Trigona fulviventris have very similar wings that could confuse the system, however these species differ in coloration of the abdomen. With some easy questions to the user, without need of training or overspending in laboratories, the system would certainly improve the detection rate by species. However, if an algorithm is as automated as possible is wanted, can add a geo-reference image can be added at the system as a new discriminator parameter. 
TABLE I PART OF THE HUGE MATRIX WITH ALL CORRESPONDENCES BETWEEN SPECIES IN THE TRAINING PHASE

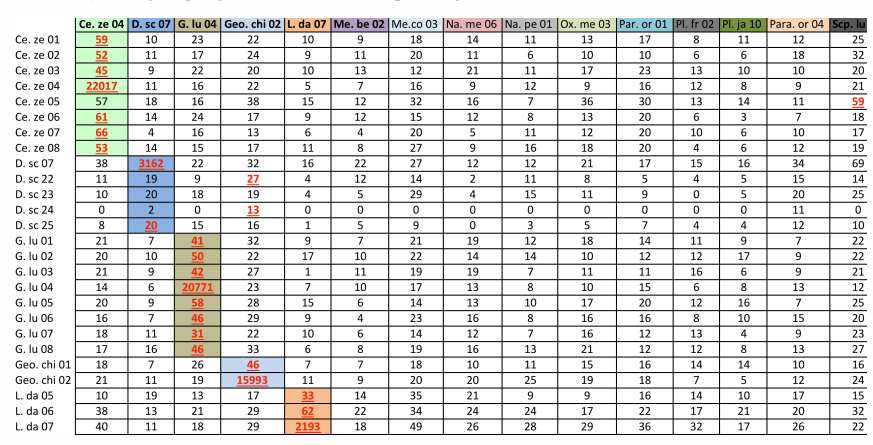

TABLE II. SPECIES SAMPLED, WRONG MATCHES AND PERCENTAGES OF SUCCESSFUL DISCRIMINATION OF COSTA RICAN NATIVE BEES

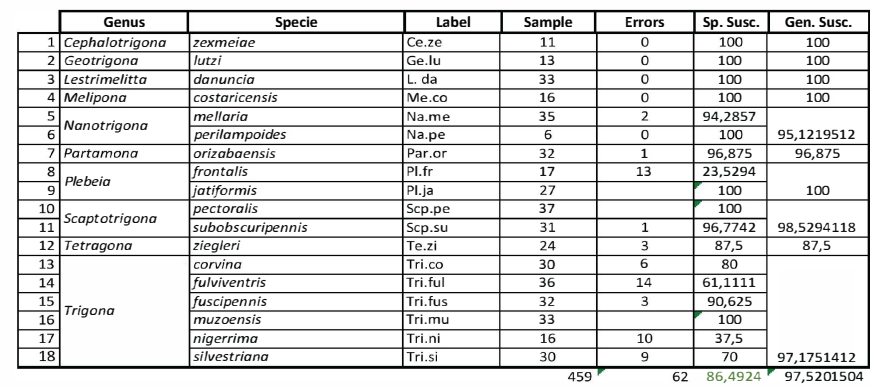

On the other hand, some of the remaining tasks are the transfer of the algorithm to a less controlled environment and promotion of the use of the tool between meliponicultors. It would be a great advance in the implementation of the algorithm in a portable device if the preprocessing can identify the bee's wing even with the presence of the body. Furthermore, with the design of an interface for the user and maintenance on a server all meliponicultors and bee researchers will have a very valuable tool.

\section{REFERENCES}

[1] Virginia Meléndez R., Laura Meneses C., Cavillo, Peter G K. (2013). Effects of Human disturbance and habitat fragmentation on stingless bees. En: Pot-Honey: A legacy of stingless bees. Patricia Vit et al. (eds), Springer New York. $629 \mathrm{p}$.

[2] Michener, C. (2000). The bees of the world. The Johns Hopkins University press.2 ed. US. 913 p.

[3] Breno M freitas, Vera Lúcia Imperatriz-Fonseca, Luis M. Medina, Astrid de Matos Peixoto Kleinert, Leonardo Galetto, Guimar Nates. Parra, J.Javier G. Quezada-Euán. (2009) Diversity, threats and conservation of native bees in the Neotropics. Apidologie. 40: 332-346

[4] Brown JFM, Paxton JR. (2009). The conservation of bees: a global perspective. Apidologie 40:410 - 416.

[5] Camargo JMF, Pedro SRM. (2007). Meliponini Lepeletier, 1836. pp. 272 - 578. In Moure JS, Urban D, Melo GAR, eds. Catalogue of bees (Hymenoptera, Apoidea) in the Neotropical Region. Sociedade Brasilera de Entomologia; Curitiba, Brasil. 1958 pp.

[6] Ricardo Ayala and Michael S. Engel. (2014). A new stingless bee species of the genus Nogueirapis from Costa Rica (Hymenoptera: Apidae). Journal of Melittology 37: 1-9.

[7] Alexandra-Maria Klein, Bernard E Vaissière, James H Cane, IngolfSteffan-Dewenter, Saul A Cunningham, Claire Kremen and TejaTscharntke. (2007). Importance of pollinators in changing landscapes for world crops. Proc. R. Soc. Bvol. 274 no. 1608 303-313

[8] Arbuckle T.; Schröder, S.; Steinhage, V. \& Wittmann, D. (2001) Biodiversity Informatics in Action: Identification and Monitoring of Bee
Species using ABIS. 15th International Symposium Informatics for Environmental Protection, Zürich, pp. 425-430

[9] Tofilski, A. (2007). Automatic measurements of honeybee wings. In MacLeod, N. (ed.), Automated Object Identification in Systematics: Theory, Approaches, and Applications, Systematics Association Special Volume, CRC Press, Boca Raton, Florida, pp 289-29

[10] Francoy, T.M., Silva, R.A.O., Nunes Silva, P., Menezes C. \& Imperatriz-Fonseca, V.L. (2007) Gender identification of five genera of stingless bees (Apidae, Meliponini) based on wing morphology. Genetics and Molecular Research 8 (1); 207-214

[11] Christopher J. Hall. (2011). Automated approach to bee identification from wing venation. Tesis de Maestría. Universidad de WisconsinMadison, USA.

[12] Breuker, C.; Gibbs, M.; Van Dongen, S.; Merckx, T. \& Van Dyck, H. (2010) The Use of Geometric Morphometrics in Studying Butterfly Wings in an Evolutionary Ecological Context Morphometrics for Nonmorphometricians, Lecture Notes in Earth 271 Sciences 124, DOI 10.1007/978-3-540-95853-6_12, C _ Springer-Verlag Berlin Heidelberg 2010

[13] Hall, M.J.R.; MacLeodb, N. \& Wardhana, A.H. (2014) Use of wing morphometrics to identify populations of the Old Worldscrewworm fly, Chrysomya bezziana (Diptera: Calliphoridae): A preliminary study of the utility of museum specimens. (C) International Atomic Energy Agency 2014. Published by Elsevier B.V. All rights reserved, pp 549555

[14] Francoy, T. M; Wittman, D.; Drauschke, M.; Muller, S ; Steinhage, V.; Bezerra-Laure, M; De Jong, D.\& Goncalves, L. (2008) Identification of Africanized honey bees through wing morphometrics: two fast and efficient procedures. Apidologie 39 (2008) 488-494

[15] Prendas J.P \& Ramírez, M. (2011) Algoritmo para la detección y seguimiento del ácaro $\mathrm{v}$. destructor en secuencia digitales de video. Tesis de licenciatura. Universidad Nacional, Costa Rica.

[16] Rafael C. Gonzalez \& Richard E. Woods. (2012) Digital Image Processing using MatLab (3rd Edition) Prentice Hall

[17] Mark Nixon (2012) Feature Extraction \& Image Processing for Computer Vision [Kindle Edition] Academic Press; 3 edition.

[18] Lowe, D. (1999) Object Recognition from Local Scale-Invariant Feature. International Conference of Computer Vision.

[19] Lowe, D. (2004) Distinctive Image Features from Scale-Invariant Keypoints. International Conference of Computer Vision.

[20] López, Y. (2013) Desarrollo de un sistema biométrico de identificación facial mediante el uso de parámetros SIFT. Proyecto de fin de Carrera. Universidad de Las Palmas de Gran Canaria, España

[21] Alguacil, A. (2009) Aplicaciones del operador SIFT al reconocimiento de objetos. Universidad Carlos III de Madrid, España

[22] Plaza, A.M. \& Zambrano, J.L. (2012) Estudio y selección de las técnicas SIFT, SURF, ASIFT de reconocimiento de imagenes para el diseño de un prototipo para dispositivos móviles. 15o Concurso de Trabajos Estudiantiles. Universidad Salesiana.

[23] Huiyu Zhoua, Yuan Yuanb, Chunmei Shi. Object tracking using SIFT features and mean shift. Computer Vision and Image Understanding. Elsevier. Volume 113, Issue 3, March 2009, Pages 345-352

[24] Plinio Moreno, , Alexandre Bernardino, José Santos-Victor.Improving the SIFT descriptor with smooth derivative filters . Pattern Recognition Letters. Elsevier. Volume 30, Issue 1, 1 January 2009, Pages 18-26

[25] Morteza Zahedi, Seyed Mahdi Salehi. License plate recognition system based on SIFT features.Procedia Computer Science Volume 3, 2011, Pages 998-1002

[26] Herrera, E., Aguilar, I., Prendas, J.P.; Figueroa, G.; Travieso C.M.; \& Ramírez, M. (2013) Photograph capture protocol for the creation of an automatic classification. En Zamora, L G (ed.) Memorias del VIII Congreso Mesoamericano de Abejas Nativas. Universidad Nacional, Heredia, Costa Rica, 26-31 Agosto; 2013. 337 p

[27] Prendas, J.P.; Figueroa, G.; Travieso C.M.; Herrera, E., Aguilar, I. \& Ramírez, M. (2013) Sistema automático de clasificación de abejas sin aguijón (Apidae: Meliponini) basado en el contorno y venación de sus alas. Propuesta y Avances. Memorias del VIII Congreso Mesoamericano de Abejas Nativas. Universidad Nacional, Heredia, Costa Rica, 26-31 Agosto; 2013. 224 p. 